\title{
Differential Cherenkov detectors: experimental technique and results
}

Mokhnachevskaya V.P.1, Egorov Y.A., Knurenko S.P., Sabourov A.V., Sleptsov I.Ye., Petrov Z.E.

$Y u$. G. Shafer Institute of cosmophysical research and aeronomy SB RAS

Yakutsk, Lenin avenue 31, Russia

E-mail: valyamokheyandex.ru

The paper provides a detailed technical description of the complex detector "Obscura". It consists of three registration points which were built around differential Cherenkov light detectors whose operation is based on a principle of "camera-obscura". Here we consider experimental techniques used for Cherenkov light registration with this type of detectors. Description is given for hardware and software parts of a system intended for previewing and recording the signal from Cherenkov detectors. Protocols of detectors calibration and measurement control are discussed.

36th International Cosmic Ray Conference -ICRC2019-

July 24th - August 1st, 2019

Madison, WI, U.S.A.

${ }^{1}$ Mokhnachevskaya 


\section{Introduction}

The differential Cherenkov detectors are working in the consist of the Yakutsk array since 2003. These detectors are located at a distance of $250-500 \mathrm{~m}$ from the center of the current Yakutsk array. This location allows the detectors to work as part of array and record shower events. One of the advantages of differential Cherenkov detectors in comparison with Cherenkov integral detectors is the substantial suppression of the noise background of the night sky. A low noise level is achieved by selecting a small field of view through the slit for each photomultiplier of photomosaic.

\section{Experimental set-up}

At this stage of the experiment, three differential Cherenkov detectors operate. These detectors based on the camera obscura consist of two parts: the camera obscura (room), photomosaic and data acquisition system. The chamber is a room with darkened walls. Over the past years, the configuration of photomosaic and slots has changed and now, finally, it looks as shown in figure 1 .

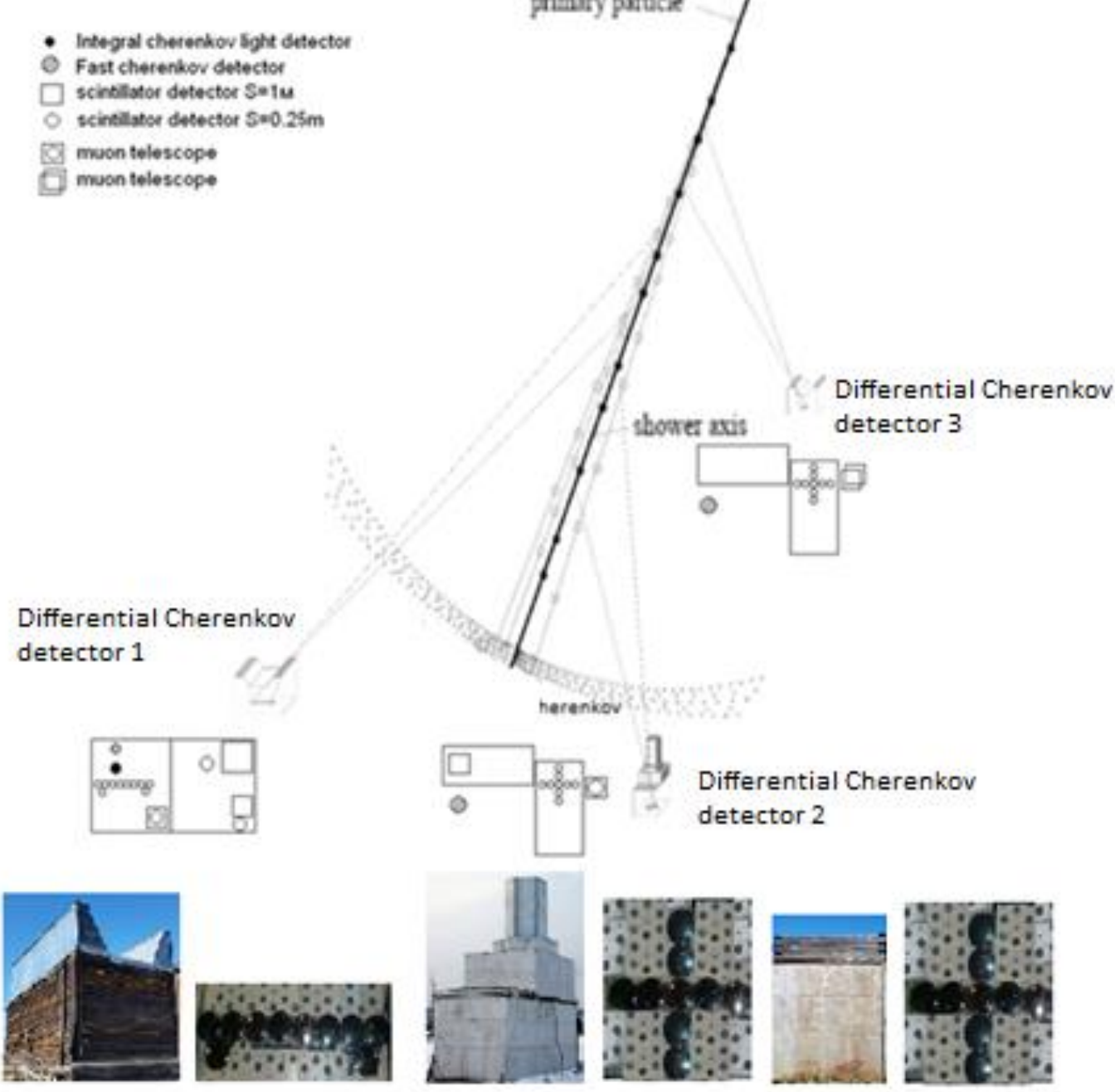

Figure 1. Differential Cherenkov detectors on basis camera obscura

Photomosaic consists of a photomultiplier line, each of the photomultiplier tubes observes a certain height of the celestial sphere through a narrow slit of the chamber. A more detailed description of the dimensions of the detectors were described in $[1,2]$. An important advantage 
detector is the ability to determine the spatial and temporal picture of the development of the Cherenkov component of EAS in the atmosphere.

In the plane of the photomosaic, the image of Cherenkov radiation manifests itself as a sequence of responses of the activated photomultipliers with different amplitudes and shapes. The level of amplitudes recorded in the image depends on the energy of the primary particle and the shower trajectory relative to the camera slits, i.e. coordinates of the shower axis in the installation plane, zenith and azimuth angles.

\subsection{Electronics and software}

To register the photomultiplier response form, computers of the Pentium enhanced reliability class are used with a backplane for PCI slots. PCI slots are equipped with La-n10m8 PCI cards with two fast 8-bit ADCs with a sampling frequency of $100 \mathrm{MHz}$ and a $2 \mathrm{MB}$ buffer memory. The system is equipped with software control of the amplification of signals from the photomultiplier outputs, each board serves 2 measuring channels. Observation points are integrated into the overall installation network. Data transmission and time synchronization of stations are carried out over the fiber-optic network (Fig. 2).

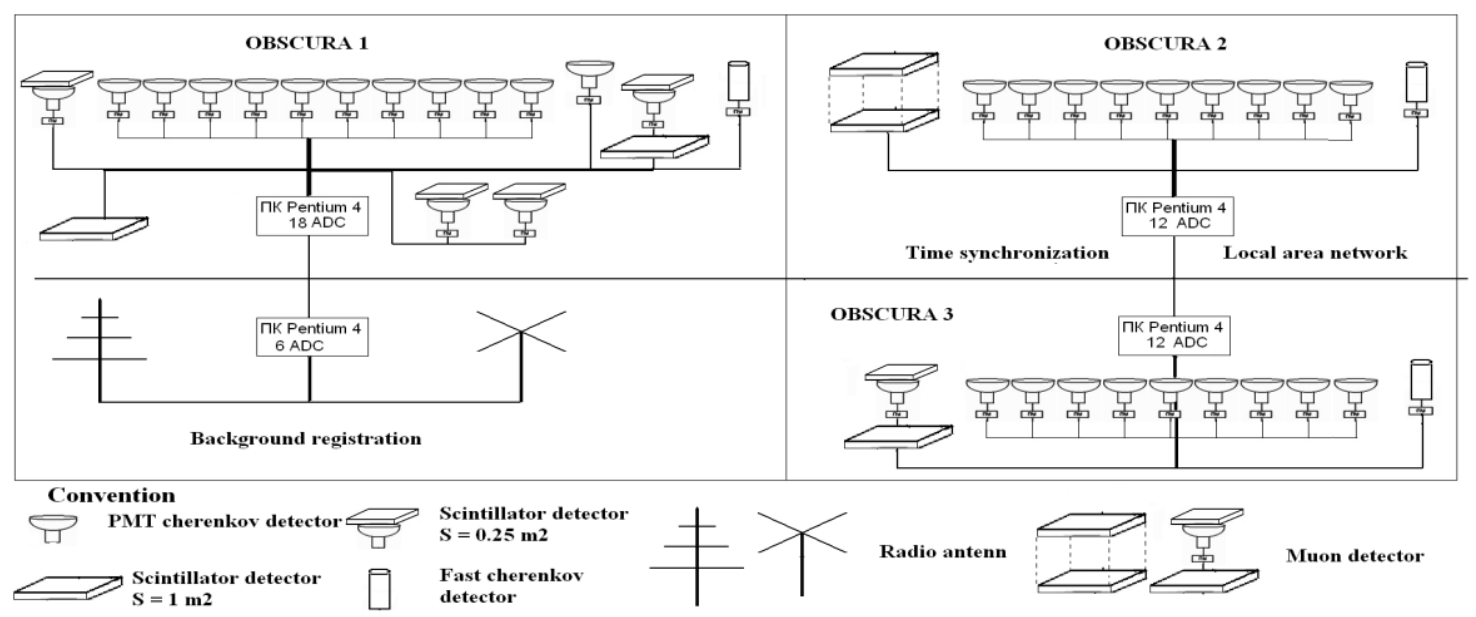

Figure 2. Block diagram of the differential Cherenkov detectors registration system

The software of each detector includes 3 components: registration of observations, preliminary analysis and registration of calibration measurements with processing. All software components work in the Windows environment and have a graphical interface to control the operation of programs. Prior to the observation session, the gain of the ADC boards common for each group is set, after which the registration program goes into the "prehistory" filling mode by the current detector counters and waiting for the master pulse. For each event along with the measurement data, date, time, sequence number of the event in the monitoring session, and gain values of the ADC boards are recorded in the file. This service information is used to match the differential Cherenkov detectors events with events on large and small Cherenkov installations.

Registration control is carried out from external master signals. To create a "master" of a large installation, it is required that the responses of the three scintillation detectors coincide and do not lie on one straight line. The "masters" of a small Cherenkov installation [3], require the coincidence of the responses of three Cherenkov integral detectors located at the vertices of equilateral triangles. This sampling system allows the recording of pulses in showers with energies above $10^{16} \mathrm{eV}$.

The registration programs start the ADC in a continuous mode and convert the signals from the photomultiplier outputs and cyclically record them into the buffer memory area called the "prehistory". The "prehistory" contains the records of ADC samples for the last $13 \mu$ s of signals, the time interval between neighboring samples being $10 \mathrm{~ns}$. After the arrival of "masters" from 
the large installation or SCI, the area of the buffer memory called "history" records color signals of the "master" type and data on the amplitude of the calibration LED, forming, in combination with the prehistory, an event frame (Fig. 3). The received frames of shower events are made into a file record and added to the initial file created automatically at the start of the observation session.

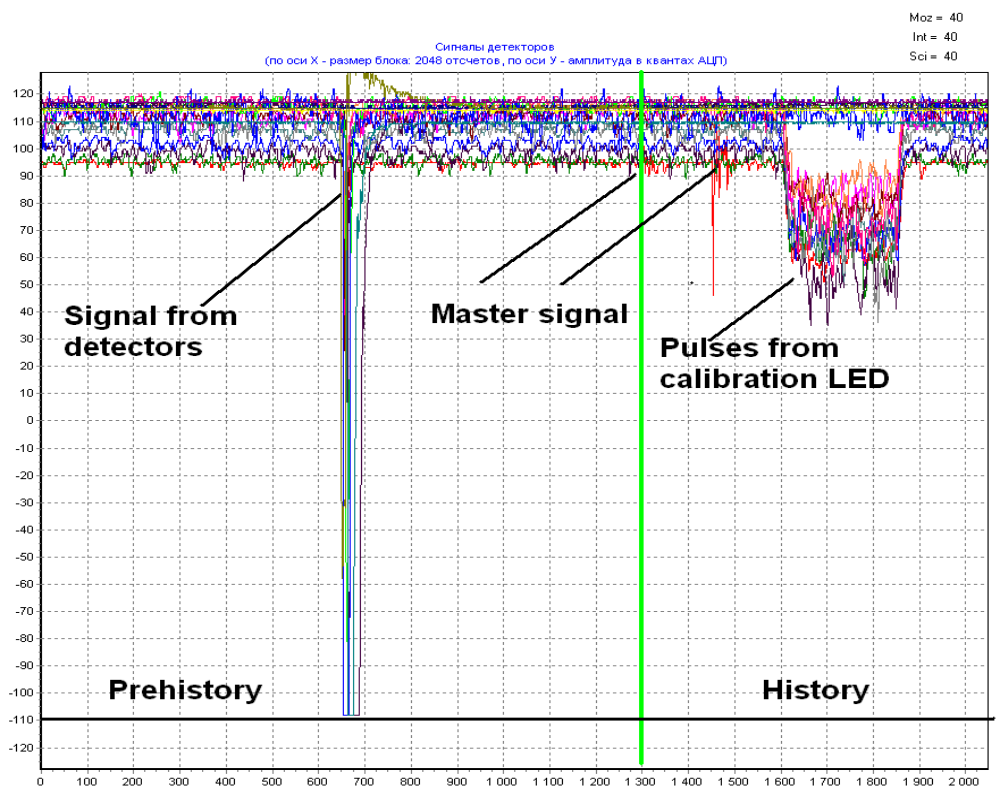

Figure 3. Air shower event registration frame

Preliminary analysis includes viewing frames on a time-extended scale, finding a useful signal in the "prehistory" and determining the parameters of the signals - amplitude, area, halfwidth and maximum moment.

\subsection{Calibration measurements}

Calibration measurements of detectors make it possible to determine the signal level from one particle of the system "PMU - preamplifier - ADC". The response of each photomultiplier is related to a known number of photons generated by the laser. The calibration diagram is shown in Figure 4. With the help of the geometric method and by taking into account the aperture of Cherenkov receivers, we restored the heights observed by each of the photomultipliers, and the parameters of the recorded pulses, including the pulse area, were attributed to these heights. In this case, the area of the pulse reflects the number of photons coming from a given height with known intensity. It is the constant intensity of laser radiation, and so the shape of the recorded pulse, which is the basis of the chosen calibration method [4]. Change in weather conditions, i.e. the conditions for the propagation of optical signals in the atmosphere, requires the introduction of corrections in the analysis of the calibration data.

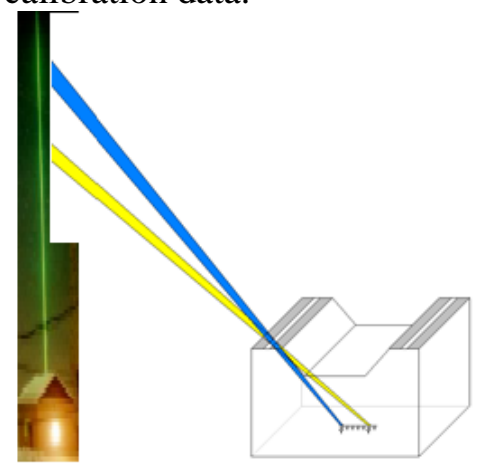


Figure 4. Diagram of Cherenkov detector calibration with the help of a laser "flash"

\subsection{Events selection and data processing}

For data processing selects all events where from 7 and more photomultiplier of photomosaic worked. Figure 5 shows the event registration in defferential Cherenkov detector 1 , where all 10 photomultiplier of photomosaic triggered. All primary data are determined by the testimony of the observation stations of the Yakutsk installation that participated in the registration of air shower: $\mathrm{x}_{0}, \mathrm{y}_{\mathrm{o}}$ - coordinates of the shower axis intersection with the installation plane, shower energy $\mathrm{E}_{0}$, shower arrival angles $\theta, \phi$, total number of charged particles $\mathrm{N} s$ at the observation level. The error in determining the coordinates of the shower axis is $\sim 15-35 \mathrm{~m}$, and the zenith angle of the direction of arrival is with an accuracy of $\sim 3-5^{\circ}$.

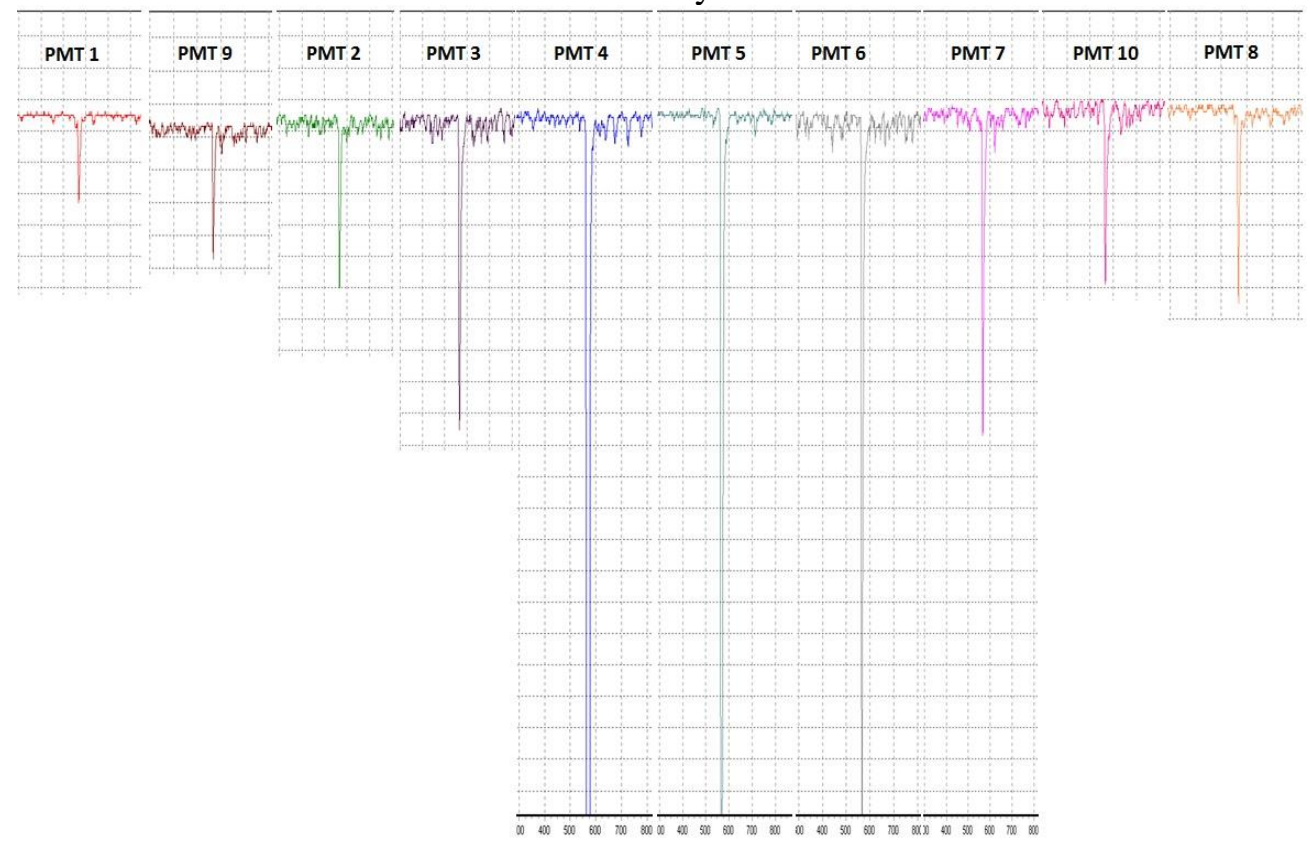

Figure 5. Pulse sweep of each photomultiplier of photomosaic of the differential Cherenkov detector 1

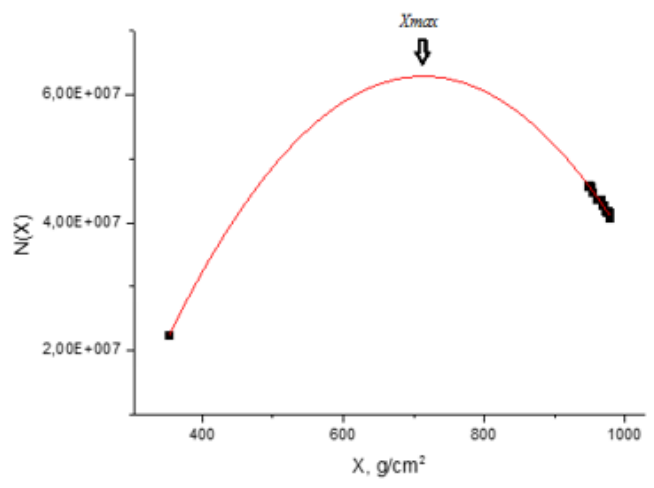

Figure 6. Dependence of the number of particles $N(x)$ on the depth of the $X$ of air shower development

According to the registration data processing program, H-heights [5] in meters are determined by each mosaic photomultiplier of the corresponding Cherenkov differential detector and are recalculated to atmosphere depth $\mathrm{X}$ in $\mathrm{g} / \mathrm{cm}^{2}$. Then, the total number of charged particles is found at the observation level from scintillation detectors of a large installation. In the future, this characteristic of air showers is used to construct the tail of the cascade curve beyond the 
maximum development of air showers $\left(\mathrm{X}_{\max }\right)$ and reconstraction the cascade curve in a wide range over the depth of the atmosphere (Fig.6).

\section{Conclusion}

Currently, work is underway to modernize the Yakutsk array. The debugging of the new version of electronic equipment of observation stations and the method of data collection and processing. Increasing the accuracy of the station's timing synchronization. This will give us a significant improvement in the accuracy of determining the parameters of air showers, localization of the shower axis in space, i.e. coordinates of the shower axis in the installation plane, the zenith and the azimuth angles. It is planned to create its own trigger, which will allow us to determine the direction of the air showers arrival independently of the larger installation. To increase the amount of the photomultiplier of photomosaic of the differential Cherenkov detector, which will give us an increase in the viewing angle of the detector and the accuracy of determining the shape of the cascade curve of the shower development in the atmosphere.

\section{References}

[1] Garipov G.K. et al. The Cherenkov Track Detector Consisting of the Yakutsk Complex EAS Array, in proceedings of ICRC2001, p.885.

[2] Egorov Y.A. et al., Cherenkov differential detector at the Yakutsk extensive air shower array, in proceedings of ICRC2017, p.462.

[3] Glushkov A.V. et al., Control and monitoring at the Yakutsk EAS array, Yakutsk: YaF S B AS USSR, 1974, p 43.

[4] Knurenko S.P., Egorov Y.A. Petrov I.S. Optic detectors calibration for measuring ultra-high energy extensive air showers Cherenkov radiation by $532 \mathrm{~nm}$ laser. Proc. SPIE 9292, 20 ${ }^{\text {th }}$ International Symposium on Atmospheric and Ocean Optics: Atmospheric Physics, 929258

[5] Mokhnachevskaya V.P. et al. $X_{\max }$ reconstruction for EAS with energy above $10^{16} \mathrm{eV}$ from responses of tracking Cherenkov detectors, in proceedings of ICRC2017, p.341. 